\title{
LIFETIME OF ELECTRONS ON THE LIQUID SURFACE OF SUPERFLUID ${ }^{4} \mathrm{He}$
}

\author{
M. ENGELHARDT, D. WEBER ${ }^{1}$ and W. SCHOEPE \\ Institut für Angewandte Physik, Universität Regensburg, 8400 Regensburg, West-Germany
}

Received 3 January 1979

Revised manuscript received 11 April 1979

The lifetime of electrons deposited on the liquid surface of ${ }^{4} \mathrm{He}$ was measured. It is smaller than $8 \mu 8$ at $0.5 \mathrm{~K}$ and, as is concluded from its temperature dependence, governed by the electronic mobility parallel to the superfluid surface.

An electron in the vapor above a ${ }^{4} \mathrm{He}$ liquid is attracted toward the liquid surface by the far-reaching image force. As the electron approaches the liquid a short-ranging repulsive force becomes effective, arising from the Pauli exclusion principle. Both forces lead to a hydrogenlike energy spectrum for the motion of electrons perpendicular to the liquid surface $[1,2]$ :

$E_{n} \approx-7.5 / n^{2}[\mathrm{~K}]$.

The motion parallel to the surface is not quantized, so that the electrons are free to move in two dimensions. Below $1 \mathrm{~K}$ only the ground state is populated. Without an electrical holding field normal to the surface the electrons will escape from their bound surface states into the vapor. This escape mechanism is thought to be governed by thermal activation leading to an increasing lifetime of the surface states when the temperature is lowered. Earlier measurements of the lifetime, however, gave the opposite results [4]: it was found to decrease when the temperature was lowered from $3.5 \mathrm{~K}$ to $1.6 \mathrm{~K}$.

It was the purpose of the present work to investigate the escape mechanism by measuring the lifetimes of the surface states in a wider temperature range. In contrast to the early experiments on lifetimes $[3,4]$, we constructed a measuring cell without moving parts (fig. 1a). The collector was connected to an electrometer and a chart recorder. With this arrangement the

\footnotetext{
1 Present address: Department of Physics, University of Idaho, Moscow, ID 83843.
}

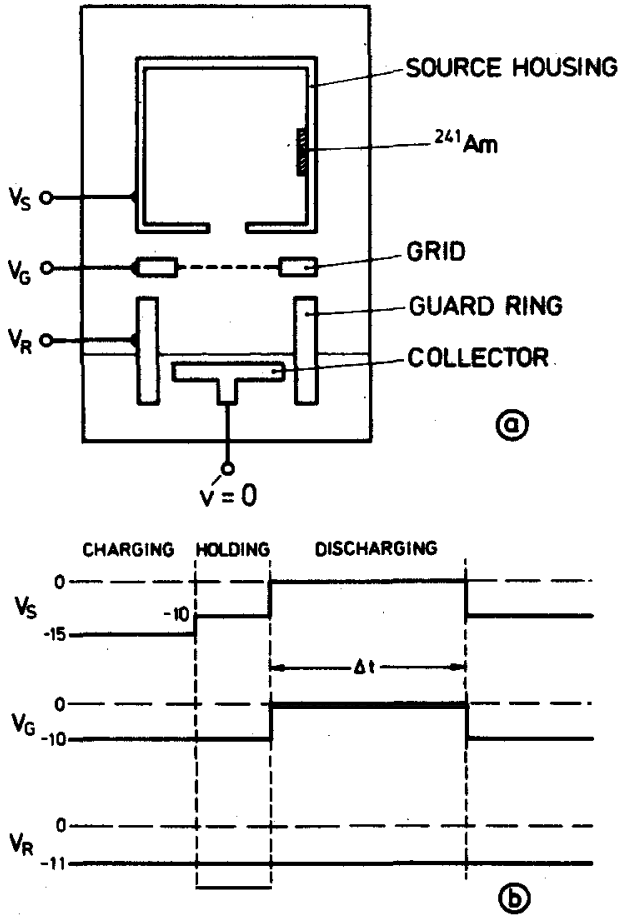

Fig. 1. (a) Measuring cell. The area of the liquid surface inside the guard ring is $2.5 \mathrm{~cm}^{2}$. The spacing between collector and grid is $0.6 \mathrm{~cm}$. (b) Time dependence of the various potentials.

amount of charge being deposited on the liquid surface is sensed by the electrometer. The guard ring prevented the electrons from escaping from the liquid surface laterally. Electrons were generated by He-gas atom ionization by $\alpha$-emitting ${ }^{241} \mathrm{Am}$. The electron current out from the source housing could sensitively 


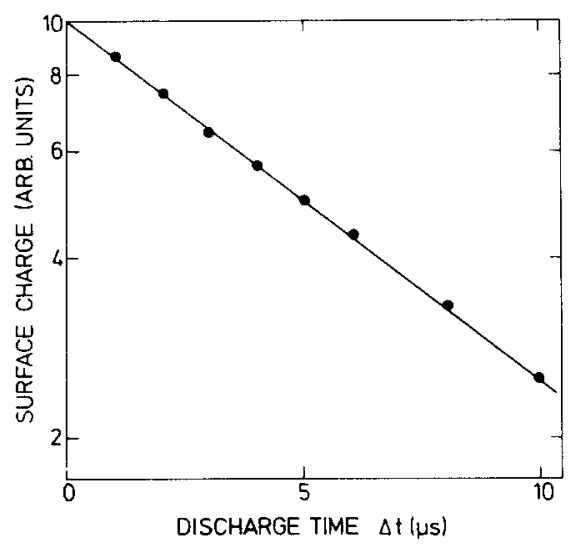

Fig. 2. Surface charge as a function of the time $\Delta t$ the holding field was switched off. $T=1.1 \mathrm{~K}$.

be regulated by the voltage between housing and grid.

${ }^{4} \mathrm{He}$ was condensed into the measuring chamber till the liquid level was in the middle of the guard ring above the collector. The liquid surface could then be charged with the source switched on. The electrometer measured the current to the surface and thus the amount of surface charge. When this current ceased indicating that the electric field between grid and collector was cancelled, no further electrons could be deposited, and the source was switched off. The charge could be held on the surface for hours without any measurable loss. Now a pulser switched off the external holding field and electrons were free to be desorbed during the pulse length $\Delta t$ (see fig. $1 \mathrm{~b}$ ).

The amount of charge that had left the surface during the time $\Delta t$ could be measured in two ways: either the current emerging from the surface was integrated or the amount of charge was measured which could be deposited until the applied field was cancelled again. Both methods gave the same results. The amount of charge on the surface as a function of pulse length $\Delta t$ was found to follow an exponential law (fig. 2):

$Q(\Delta t)=Q_{0} \exp (-\Delta t / \tau)$.

The lifetime $\tau$ was thus determined at different temperatures (fig. 3). It was found to be independent of the applied field, i.e. independent of $Q_{0}$ which was the maximum amount that could be stored in a given field. $Q_{0}$ was typically between $3 \times 10^{6} \mathrm{e} / \mathrm{cm}^{2}$ and 3 $\times 10^{7} \mathrm{e} / \mathrm{cm}^{2}$.

The measured temperature dependence of the lifetime is in striking contrast to what we had expected

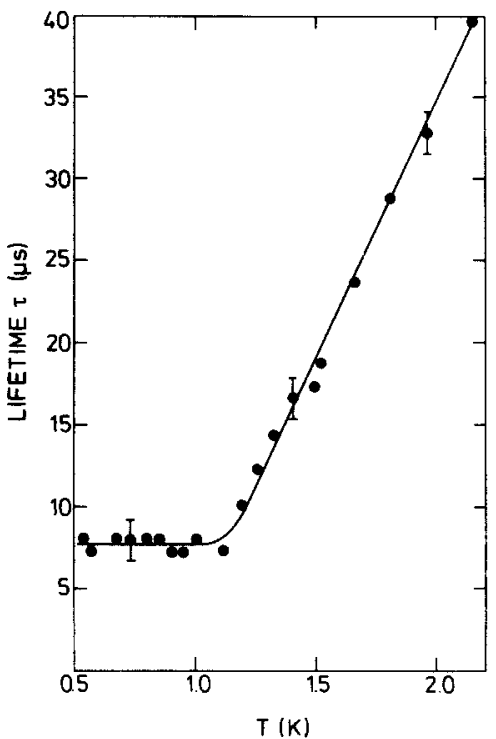

Fig. 3. Dependence of the lifetime $\tau$ on temperature.

assuming a thermally activated desorption mechanism. It decreases with decreasing temperature similar to earlier observations [4] and levels off below $1.1 \mathrm{~K}$. In view of these peculiar results we have checked whether the electrons are lost by some other mechanisms, e.g. transients coupled capacitively to the confining guard ring ${ }^{\ddagger 1}$ and have found none. In addition time of flight effects cannot have falsified our data because they were small compared to $\tau$ and also lead only to an irrelevant shift of the time scale in fig. 2. A possible explanation for the observed temperature dependence might be the following: Since the potential of the guard ring and the electric field above the liquid produce maximum electronic density in the middle above the collector, electrons from this area are desorbed first when the discharging period begins. They are restored by electrons from outer regions, which drift to the center to be emitted there.

A comparison of the temperature dependences of the lifetime and the electronic mobility [5] on the liquid surface suggests that the measured lifetime includes a drift-time $\tau_{\|}$of electrons parallel to the sur-

\#1 The transients which were of the order of $1 \mathrm{~V}$ had died out within less than $0.5 \mu$ s. Pulses of this length were found to be too short to remove any measurable surface charge. We are grateful to Dr. C.C. Grimes for calling our attention to these checks. 
face. The levelling off below $1 \mathrm{~K}$ could be identified with the regime where the electron motion is governed by ripplon scattering whereas the steep increase of lifetime (decrease in mobility) above $1 \mathrm{~K}$ can qualitatively be ascribed to gas atom scattering becoming more effective at higher temperatures. The measured lifetime then is the sum $\tau=\tau_{\perp}+\tau_{\|}$, where $\tau_{\perp}$ is the lifetime for desorption. Since we cannot find an increase of $\tau$ below $1 \mathrm{~K}$, where $\tau_{\|}$is nearly constant [5], we conclude that at our lowest temperature the lifetime of the electrons is still determined by their diffusion time and that the proper lifetime for desorption $\tau_{\perp}$ is still shorter than $8 \mu \mathrm{s}$ at $0.5 \mathrm{~K}$.

We have extended these experiments to electrons on thin helium films by lowering the liquid level below the collector. In this case the binding energy of the surface states and hence the lifetime $\tau_{\perp}$ should be considerably larger. In addition any motion of the electrons parallel to the surface should be much slower because of the "dimple" which is believed to exist below each electron in this case [6]. Thus a much longer lifetime was expected depending on temperature and film thickness. In general we obtained similar exponential curves for $Q(\Delta t)$, with $\tau$, however, being of the order of seconds. The amount of charge $Q_{0}$ which could be stored on the film was by an order of magnitude bigger in this case. Unfortunately the results were rather irreproducible which prevented us from obtaining a consistent set of data.

Obviously, further experimental work is required for an understanding of the desorption mechanism of electrons from the free surface of liquid helium.

We acknowledge valuable discussions with Profs. K.F. Renk and F. Bridges.

Note added. After this paper was submitted a similar experiment in a different geometry was published by Iye et al. [7] . Though the time dependence of the signal obtained by these authors is quite different from our purely exponential decay of the surface charge, their results roughly agree with ours above $1.2 \mathrm{~K}$. Between $1.1 \mathrm{~K}$ and $0.9 \mathrm{~K}$, however, their data are in striking contrast to ours. At present, the reason for this discrepancy is not clear; it may be caused by the rather different experimental conditions, e.g. the electric field configuration at the liquid surface.

\section{References}

[1] M.W. Cole and M.H. Cohen, Phys. Rev. Lett. 23 (1969) 1238.

[2] V.B. Shikin, Sov. Phys. JETP 31 (1970) 936.

[3] R. Williams, R.S. Crandall and A.H. Willis, Phys. Rev. Lett. 26 (1971) 7.

[4] R.S. Crandall and R. Williams, Phys. Rev. A5 (1972) 2183.

[5] F. Bridges and J.F. McGill, Phys. Rev. B15 (1977) 1323.

[6] L.M. Sander, Phys. Rev. B11 (1975) 4350.

[7] Y. Iye et al., J. Low Temp. Phys. 34 (1979) 539. 